\title{
An arterial pressure waveform that told a story beyond the blood pressure
}

\author{
Ragesh Panikkath MD, Deepa Panikkath MD
}

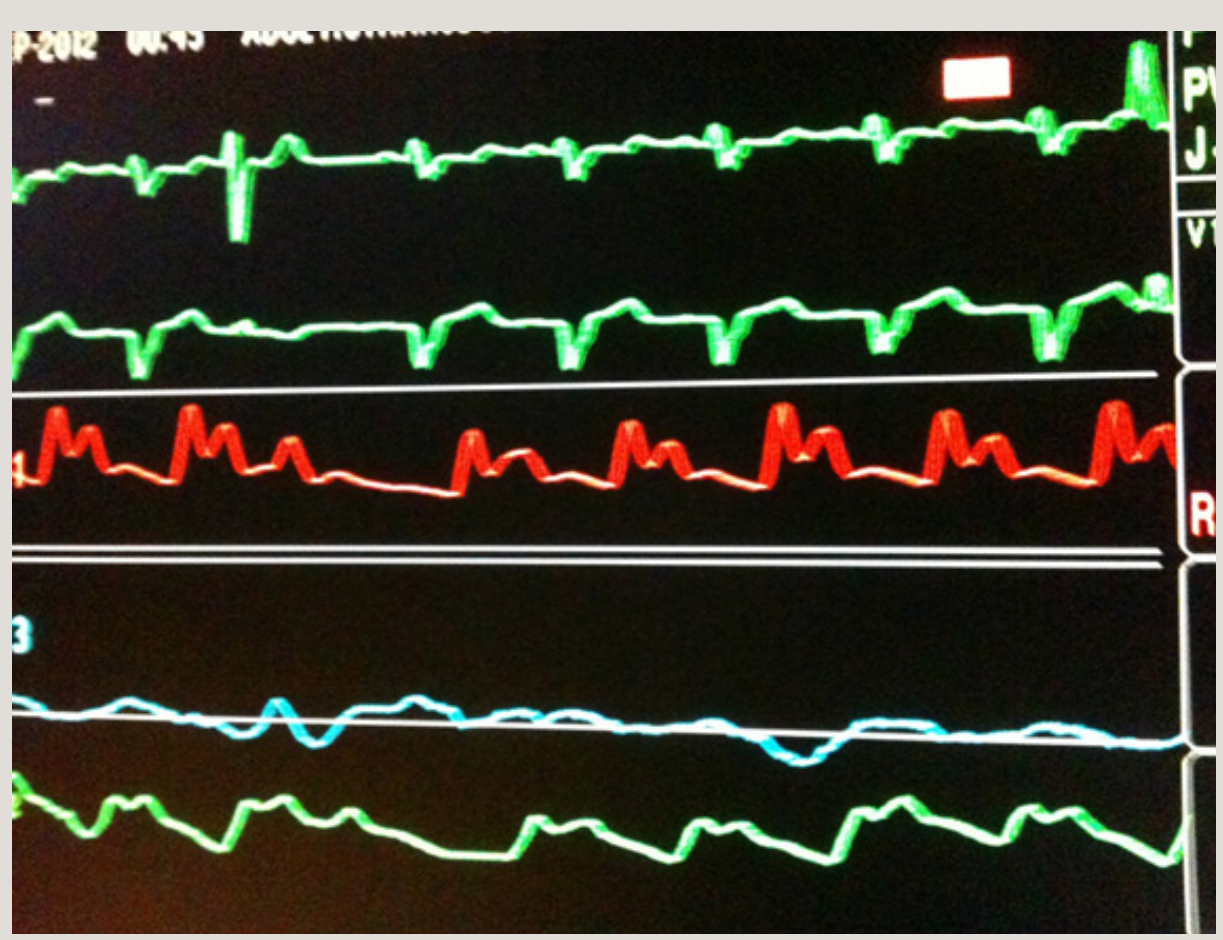

Figure Continuous ECG tracing (in green) and the arterial pressure tracing (in red). The spike and dome contour of the arterial pressure tracing is evident. The ventricular ectopic beat (3rd beat in the tracing) has only the spike pattern in the arterial pressure tracing. The first post ectopic beat shows reduced systolic pressure and pulse pressure which is also evident even in the 2nd post ectopic beat. This finding in the first post ectopic beat is known as the Brockenbrough-Braunwald-Morrow phenomenon. Similar findings although less prominent can also be seen in the pulse oximetry tracing (last tracing from bottom in the image in green color).

A 60-year-old man was admitted to the intensive care unit after a resuscitated out-of-hospital cardiac arrest. He was hemodynamically stable and had a normal mental status. His arterial wave form had a "spike and dome" pattern (Figure). However, the ventricular ectopic beat has only one component possi-

Corresponding author: Ragesh Panikkath MD

Contact Information: ragesh.panikkath@ttuhsc.edu DOI: 10.12746/swrccc 2014.0206.072 bly due to differences in ventricular activation during the ectopic beat. The recording also shows that the systolic pressure and pulse pressures are lower than the baseline in the post ectopic beat. These changes are also present in the few beats following the ectopic beat. The reduction of systolic and pulse pressure in the post ectopic beat was first described in 1961 in patients with hypertrophic cardiomyopathy ( $\mathrm{HCM}$ ) and has been called the "Brockenbrough-Braunwald-Morrow phenomenon". 1 Similar findings have been described in patients with valvular aortic stenosis. Our 
patient underwent an echocardiogram, which showed asymmetric septal hypertrophy and severe left ventricular outflow tract obstruction consistent with hypertrophic obstructive cardiomyopathy.

The spike and dome pattern is due to the rapid rise in aortic pressure during the early systole (due to the percussion wave) followed by a mid-systolic drop followed by the secondary or tidal wave. The mid systolic drop in amplitude of the pulse wave is due to a decrease in forward flow related to mid systolic obstruction which is in turn caused by the systolic anterior motion of the anterior mitral leaflet. The early rapid ejection creates a Venturi effect, which sucks the anterior mitral leaflet and the chordae into the left ventricular outflow tract. The late peak (dome) occurs when the valve leaflets return to their original position when the left ventricular outflow tract obstruction reverses.

Ectopic atrial and ventricular beats are associated with a prolonged compensatory pause. During this pause, more extracellular calcium enters the cardiac muscle cells and gets stored in the sarcoplasmic reticulum. Increased concentrations of calcium in myocardial cells cause hypercontractility. ${ }^{2}$ This leads to a higher systolic and pulse pressure in normal hearts. ${ }^{3}$ However, in patients with hypertrophic cardiomyopathy, vigorous contraction increases the systolic obstruction and lowers systolic pressure and pulse pressure in the post ectopic beat.

Author affiliation : Ragesh Panikkath and Deepa Panikkath are internal medicine residents at TTUHSC in Lubbock.

Submitted: $1 / 31 / 2014$

Accepted: $3 / 20 / 2014$

Reviewers: Aliakbar Arvandi MD

Conflict of Interest: None

Published electronically: 4/15/2014

\section{References}

1. Brockenbrough EC, Braunwald E, Morrow AG. A hemodynamic technic for the detection of hypertrophic subaortic stenosis. Circulation 1961; 23: 189-94.

2. Marengo FD, Marquez MT, Bonazzola P, Ponce-Hornos JE. The heart extrasystole: an energetic approach. Am J Physiol. 1999;276:H309-16.

3. Adawi S, Asmer I, Merdler A, Shiran A. Echocardiographic Brockenbrough-Braunwald-Morrow sign. Eur J Echocardiogr. 2011;12:E12. 